\title{
A New System for Sodium Flux Growth of Bulk GaN Part II: in situ Investigation of Growth Processes
}

\author{
Paul Von Dollen ${ }^{a}$, , Siddha Pimputkara, Mohammed Abo Alreesha, Shuji Nakamura ${ }^{a}$ \\ and James S. Speck ${ }^{a}$
}

${ }^{a}$ Materials Department, University of California, Santa Barbara 93106-5050

*Corresponding author.

Email-address: paul_vondollen@engineering.ucsb.edu (P. Von Dollen)

\begin{abstract}
We report recent results of bulk GaN crystal growth using the sodium flux method in a new crucible-free growth system. We observed a (0001) Ga face (+c-plane) growth rate $>50 \mu \mathrm{m} / \mathrm{hr}$ for growth at a $\mathrm{N}_{2}$ overpressure of $\sim 5 \mathrm{MPa}$ and $860{ }^{\circ} \mathrm{C}$, which is the highest crystal growth rate reported for this technique to date. Omega X-ray rocking curve ( $\omega$-XRC) measurements indicated the presence of multiple grains, though full width at half maximum (FWHM) values for individual peaks were $<100$ arcseconds. Oxygen impurity concentrations as measured by secondary ion mass spectroscopy (SIMS) were $>10^{20}$ atoms $/ \mathrm{cm}^{3}$. By monitoring the nitrogen pressure decay over the course of the crystal growth, we developed an in situ method that correlates gas phase changes with precipitation of $\mathrm{GaN}$ from the sodium-gallium melt. Based on this analysis, the growth rate may have actually been as high as $90 \mu \mathrm{m} / \mathrm{hr}$, as it would suggest GaN growth ceased prior to the end of the run. We also observed gas phase behavior identified as likely characteristic of GaN polynucleation.
\end{abstract}

Keywords: A1. Na flux method; A1. Growth rate; A2. Growth from high temperature solutions; A2. Sodium flux growth; B1. Nitrides; B1. III-Nitrides; B1. Bulk GaN

\section{Introduction}

Substrates derived from bulk gallium nitride $(\mathrm{GaN})$ crystals have been identified as a key enabling technology for next-generation optoelectronic and power devices. Among the techniques currently used to fabricate bulk GaN, the sodium flux method has attracted interest[1] due to the demonstrated high crystal quality[2] and more mild process conditions compared to alternatives such as hydride vapor phase epitaxy (HVPE)[3] or ammonothermal[4, 5] techniques. Both of these 
methods have received much more technological development than the sodium flux technique, which can be considered immature in comparison.

The sodium flux method involves precipitation of GaN from a melt containing sodium and gallium exposed to an overpressure of nitrogen gas.[6] Typical process pressures are in the range 3.5-5 $\mathrm{MPa}$, so in situ investigation of the growth environment or growth surface is difficult. There are only a few reports aimed at elucidating the sodium flux growth mechanism and growth kinetics.[7-9] This is in contrast to many investigations of the thermodynamics, chemistry, fluid dynamics, etc., reported for HVPE[10, 11] and ammonothermal methods,[12-16] respectively.

Growth rate improvement is another nascent topic for the sodium flux method, but is critical from an economic standpoint. The highest reported growth rates for the sodium flux method are between 40 and $46 \mu \mathrm{m} / \mathrm{hr},[17,18]$ which are much less than for HVPE[19] and similar to the highest reported values for the ammonothermal method.[20]

This paper reports recent bulk GaN crystal growth efforts using a new sodium flux system which we described in a previous publication.[21] We designed and operated the growth reactor to be able to monitor the growth process in situ using the reactor pressure decay. The system was designed to provide control over critical crystal growth parameters, such as seed position and temperature, which should then translate into improved GaN crystal growth.

\section{Experimental Details}

\subsection{Growth Runs}

GaN crystal growth was carried out using a carbon-added sodium-gallium melt contained within a crucible-free molybdenum capsule (Gen III) system. The capsule was situated inside an internally-heated stainless steel reactor pressurized with $\mathrm{N}_{2}$ gas. The growth system and experimental procedures have been detailed in Part I of this paper.[21] A summary of the key process parameters is included in Table 1.

\begin{tabular}{|ccccccc|}
\hline $\begin{array}{c}\text { Melt Composition } \\
(\mathbf{m o l} \%)\end{array}$ & $\begin{array}{c}\text { Gallium } \\
(\mathbf{m m o l})\end{array}$ & $\begin{array}{c}\text { Sodium } \\
(\mathbf{m m o l})\end{array}$ & $\begin{array}{c}\text { Carbon } \\
(\mathbf{m m o l})\end{array}$ & $\begin{array}{c}\text { Peak } \\
\text { Pressure } \\
(\mathbf{M P a})\end{array}$ & $\begin{array}{c}\text { Dwell } \\
\text { Temperature } \\
\left({ }^{\circ} \mathbf{C}\right)\end{array}$ & $\begin{array}{c}\text { Dwell } \\
\text { Time } \\
(\mathbf{H r s})\end{array}$ \\
\hline \hline Ga: 19, Na: 80.5, C0.5 & 38 & 165 & 1 & 5.2 & 860 & 134 \\
\hline
\end{tabular}

Table 1. Summary of growth parameters.

The melt constituents of sodium, gallium and carbon were added to the capsule in an $\mathrm{N}_{2}$ filled glove box $\left(\mathrm{O}_{2}\right.$ and $\left.\mathrm{H}_{2} \mathrm{O}<1 \mathrm{ppm}\right)$. The nominal gallium concentration was $19 \mathrm{~mol} \%$ with sodium at $80.5 \mathrm{~mol} \%$. Carbon, at $0.5 \mathrm{~mol} \%$ of the total melt, was used to suppress parasitic nucleation. The sodium was melted and solidified in the capsule and around the seed prior to addition of the gallium. The melt volume was nominally $5.5 \mathrm{~cm}^{3}$, corresponding to an estimated melt height of $1 \mathrm{~cm}$, or $\sim 3$ 
$\mathrm{mm}$ below the top of the vertically mounted seed. The amount of each respective melt constituent is also included in Table 1 and a schematic of the growth configuration is shown in Figure 1.

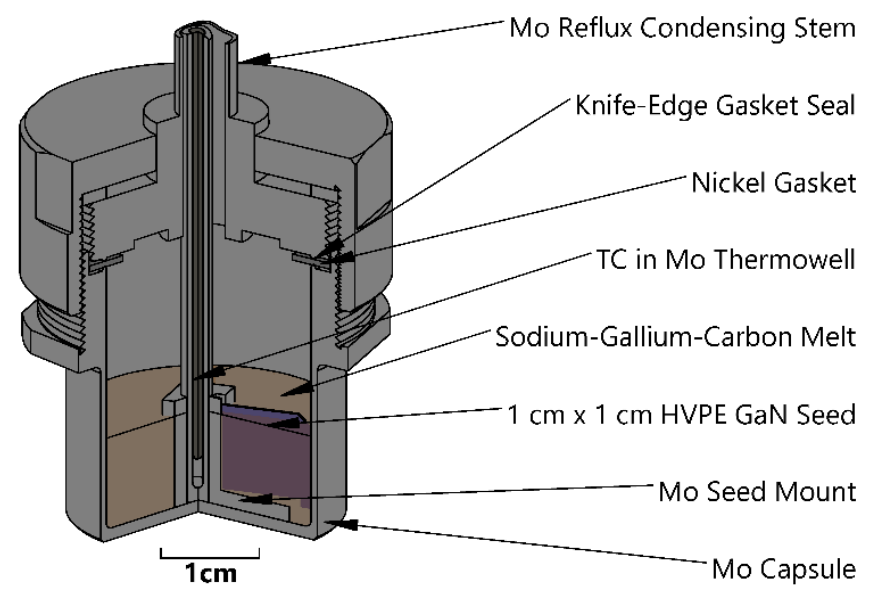

Figure 1. Schematic of run setup showing seed mounted vertically in molybdenum capsule with both (0001) Ga and (0001) $N$ faces exposed.

We previously reported the phenomenon of sodium and gallium migration during the run due to evaporation and re-condensation outside the melt, which must affect the actual melt composition since the rates are unequal.[21] This "offset" is naturally system-dependent, as it is determined by the volume accessible to the vapor as well as the temperature distribution. Based on post-run measurements, the loss of sodium from the melt case increased the gallium concentration by 1-2 $\mathrm{mol} \%$ from the initial or nominal value, while as much as $8 \%$ of the initial gallium may have been lost due to alloying with the nickel gasket during the course of the run.

A $1 \mathrm{~cm} \times 1 \mathrm{~cm} \times \sim 350 \mu \mathrm{m}$ thick $c$-plane substrate derived from an HVPE-grown bulk GaN boule (NanoWin, Suzhou, China) was mounted vertically in a molybdenum fixture (seed mount) to serve as a seed. The seed mount was located near the center of the melt with the molybdenum closed-ended thermowell in close proximity. The double-side polished seed had a specified dislocation density of $<5 \times 10^{5} \mathrm{~cm}^{-2}$ and free carrier concentration $<5 \times 10^{16} \mathrm{~cm}^{-3}$.

The capsule was sealed using a polished nickel gasket (Swagelok) deformed between two opposing knife edges. The capsule was then loaded into a stainless steel pressure vessel, which was sealed with a Teflon gasket. Following loading and sealing, the reactor was filled with $2.76 \mathrm{MPa}$ of $\mathrm{N}_{2}$ (Research Grade, Praxair) and placed inside a $\sim 1 \mathrm{~m}^{3}$ containment vessel enclosure. To reduce the effect of diurnal temperature fluctuations on the pressure inside the reactor, the enclosure ambient was maintained at a constant temperature by separate heaters. Pressure in the reactor was monitored during the course of the run using a strain gage-based transducer (Honeywell model STJE) accurate to within $\pm 3.4 \mathrm{kPa}$. The melt temperature was controlled to within $\pm 0.5^{\circ} \mathrm{C}$ of the target value through a Type $\mathrm{K}$ thermocouple located inside the thermowell submerged in the melt. The thermocouple junction was within $\sim 2.5 \mathrm{~mm}$ of the melt bottom. 
Since the reactor was internally heated, the $\mathrm{N}_{2}$ gas within the reactor was not isothermal, with a typical drop of $\sim 700^{\circ} \mathrm{C}$ between the melt and the reactor outer wall. To allow estimation of the average or effective gas temperature, a thermocouple was located within the reactor pressure head. This thermocouple also provided protection against thermal overshoot. The reactor was heated to $860^{\circ} \mathrm{C}$ over a period of 6 hours. Following a dwell of $\sim 140$ hours at $860^{\circ} \mathrm{C}$, the reactor was cooled down over 2 hours, vented, and opened.

After the run, the seed was recovered by soaking the capsule contents in a mixture of isopropyl alcohol (IPA) and water to neutralize and safely dissolve residual sodium. We took care to begin at a low concentration of water ( $5 \mathrm{vol} \%)$ to prevent any violent reaction and then gradually increased the proportion of water to completely consume the residual sodium. Following recovery, the crystal was characterized without any etching or any other post-run surface preparation.

The grown crystal was characterized using X-ray omega rocking curve $(\omega-\mathrm{XRC}$, Panalytical MPD Pro) measurements about the $\mathrm{GaN}(0002)$ reflection as well as secondary ion mass spectroscopy (SIMS, Cameca IMS 7F) depth profiles into the grown material. The $\omega$-XRC spot size was $10 \mathrm{~mm} x$ $\sim 400 \mu \mathrm{m}$ using $\mathrm{CuK} \alpha_{1}$ radiation. For the SIMS analysis, relative sensitivity factors (RSFs) previously calculated at UCSB were used to determine impurity concentrations for all elements except sulfur. RSF values for sulfur were estimated using literature tables compiled for GaAs,[22] as this values was not known for GaN. Comparison of estimated RSF values to measured values potential underestimation of the sulfur concentration of up to an order of magnitude. Error in the SIMS measurements was also introduced due to non-uniform sputtering of rough as-grown surfaces as well as the use of offset voltages for some isotopes.

\section{Results and Discussion}

\subsection{Crystal Characterization}

The crystal exhibited predominantly (0001) Ga face growth and was black and opaque in appearance. One hillock of $\sim 1 \mathrm{~mm} \times 4 \mathrm{~mm} \times 1 \mathrm{~mm}$ height formed on the (0001) N face. On the (0001) Ga face, there was evidence of skeletal or hopper growth of outer $\{10 \overline{1} 1\}$ edges initially, followed by fill-in growth on the interior. Such growth, also known as the Berg effect, is generally a sign of large gradients in supersaturation, likely due to poor melt homogeneity. Both the black color and skeletal growth are similar to previous reports for the sodium flux method grown without additives such as calcium and lithium.[23-25] An image of (0001) Ga face growth is shown in Figure 2a. The crystal cracked during post-run removal from the seed mount. 

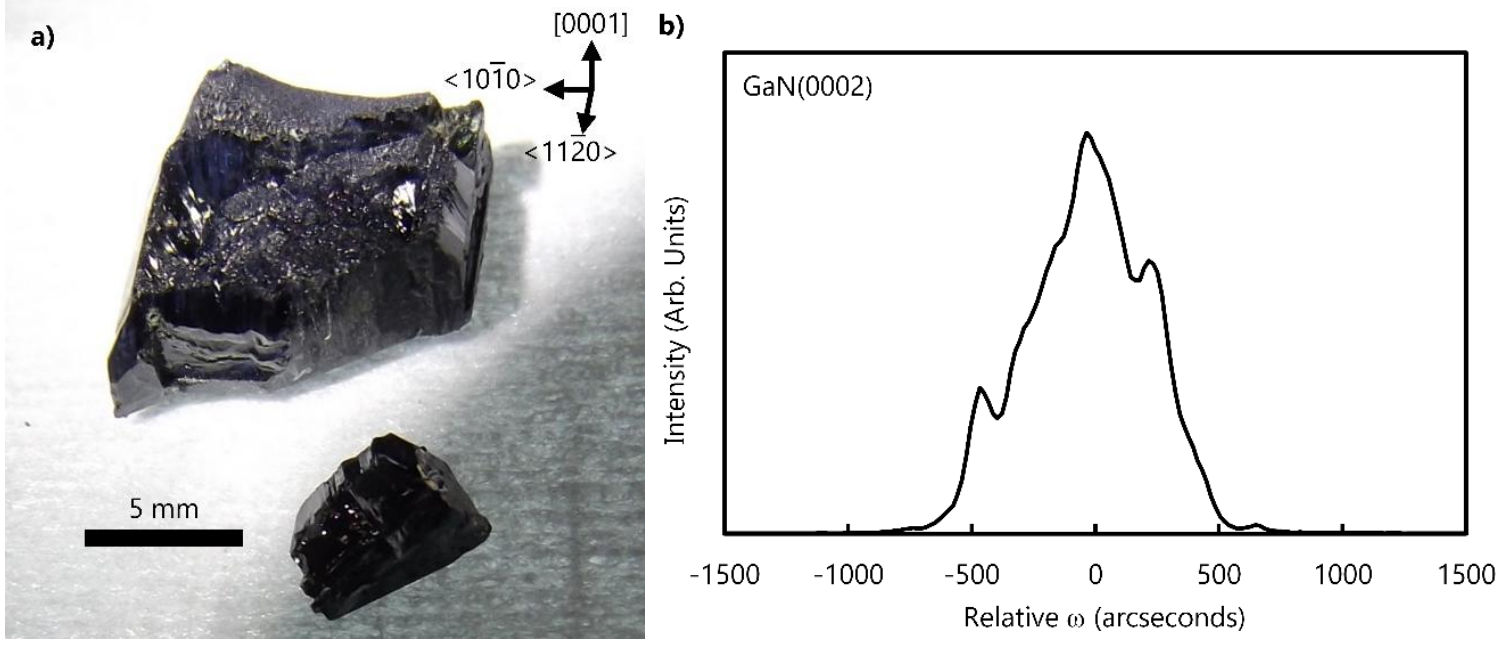

Figure 2. a) Image of grown GaN crystal. The crystal cracked during removal from the seed mount. b) On-axis GaN(0002) $\omega$-XRC spectra showing multiple peaks.

The grown crystal surface morphology was quite rough, with distinct macroscopic growth zones apparent. The total grown crystal thickness (excluding the seed thickness) ranged from 3.67 to $4.5 \mathrm{~mm}$. Except for the appearance of $\{10 \overline{1} 1\}$ facet edges, the surface did not correspond to any particular crystallographic features to the naked eye. Deep pits were present at the interior of the crystal, consistent with incomplete fill-in following hopper crystal formation, but it is also possible the areas of no growth occurred due to poor nucleation or surface contamination. Polycrystalline GaN (PolyGaN) deposition was evident on the top of the seed mount as well as in the form of a ring on the inside of the capsule near the top of the nominal melt height.

Figure $2 \mathrm{~b}$ shows the on-axis $\mathrm{GaN}(0002) \omega$-XRC from the crystal grown in Figure $2 \mathrm{a}$. Multiple peaks are apparent in the $\omega$-XRC, indicating the presence of multiple grains within the sample. The FWHM value across the multiple peaks was $~ 575$ arcseconds, while individual peaks were as narrow as $\sim 80$ arcseconds. In any case, the grown crystal quality was worse than the seed, as the measured FWHM values for the as received HVPE GaN seed crystals were 20-50 arcseconds. It is unclear if the multiple grains were due to poor initial nucleation or developed later, possibly as a consequence of the high growth rate or non-uniformities during growth.

The oxygen concentration from SIMS measurements was $>10^{20}$ atoms $/ \mathrm{cm}^{3}$, greater than reported previously for the sodium flux method.[26] The reason for the elevated oxygen level is unclear, but may be related to the loading and handling procedures. The molybdenum concentration was high, $\sim 5 \times 10^{19}$ atoms $/ \mathrm{cm}^{3}$, and likely due to ready incorporation of molybdenum dissolved in the melt from the capsule walls. This could be mitigated through the use of coatings or crucibles if molybdenum causes deleterious effects in GaN. Sodium, sulfur, aluminum, titanium, chromium and iron concentrations were all $<10^{19}$ atoms $/ \mathrm{cm}^{3}$. 


\subsection{Pressure Decay and Growth Rate}

The pressure curve peaked at $\sim 15$ hours into the run and then decayed to an asymptotic value of $\sim 5 \mathrm{MPa}$, with no major decrease evident for the last $\sim 40$ hours of the run. The lack of decrease in the pressure curve after $\sim 100$ hours suggested that leakage was negligible and that formation of GaN was the only nitrogen-consuming process. The pressure and temperature profile for the entire run duration is shown in Figure 3.

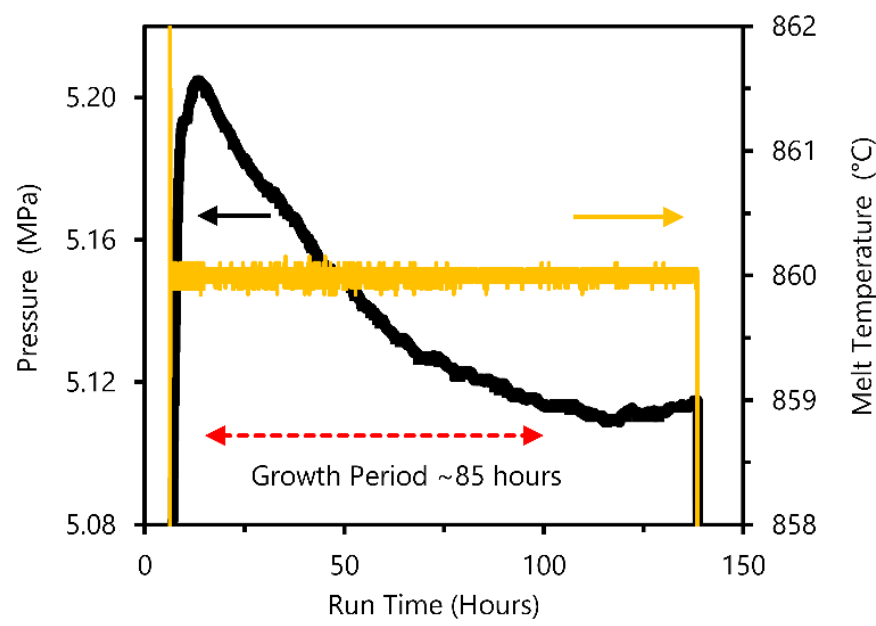

Figure 3. Pressure and temperature profile showing saturation of the pressure decay after 100 hours.

Based on this, the growth rate was calculated by dividing the total growth thickness (3.7-4.5 $\mathrm{mm})$ by the time between the peak pressure ( $\sim 15$ hours) and before the pressure curve saturated $(\sim 100$ hours), or $\sim 85$ hours, for a growth rate of $43-52 \mu \mathrm{m} / \mathrm{hr}$. This is the highest reported GaN growth rate for the sodium flux method to date.

Based on the weight and thickness of the grown crystal, the estimated gallium conversion from the melt to $\mathrm{GaN}$ was $>90 \%$. Calculation of gallium utilization was complicated by gallium uptake of the nickel gasket, as confirmed using energy-dispersion X-ray spectroscopy (EDX). Postrun weight change measurements indicated that as much as $\sim 8 \%$ of gallium from the melt was incorporated in the gasket,[27] for a total gallium utilization of $\sim 98 \%$. Such a high gallium utilization was consistent with the observed minute quantity of free gallium remaining after the run. The gasket swelled by $\sim 20 \%$ in thickness everywhere exposed to the melt, i.e. inside the knife-edge seal. The rate or trajectory of gallium uptake by the gasket was impossible to determine, but likely decreased with decreasing concentration in the melt.

\subsection{Insights from the Pressure Curve Decay}

Along with determining a more accurate growth time, we used the pressure decay during the run to make some initial investigations into the details of the growth process. To account for pressure fluctuations due to temperature changes of the $\mathrm{N}_{2}$ gas in the reactor, the pressure curve shown in Figure 3 was converted to a molar $\mathrm{N}_{2}$ curve by applying the ideal gas law 


$$
n=\frac{P V}{R T^{E f f}}
$$

where $n$ is the number of moles of $\mathrm{N}_{2}$ in the reactor, $P$ is the reactor pressure measured by the transducer, $V$ is the reactor free volume and $T^{E f f}$ is the average or effective $\mathrm{N}_{2}$ gas temperature. The free volume was estimated based on the specified naked reactor volume and previous measurements with similar configurations, and effects of thermal expansion, pressurization strain and molar volume changes during crystallization were neglected. We also neglected to account for changes in sodium vapor pressure due to changes in melt composition. We calculated an effective $\mathrm{N}_{2}$ gas temperature by interpolating between the melt temperature and reactor head temperature through

$$
T^{E f f}=T^{\text {Head }}+g\left(T^{\text {Melt }}-T^{\text {Head }}\right)
$$

where $T^{\text {Head }}$ is the reactor head temperature, $T^{\text {Melt }}$ is the melt temperature (controlled) and $g$ is a fitting factor which can be determined empirically.

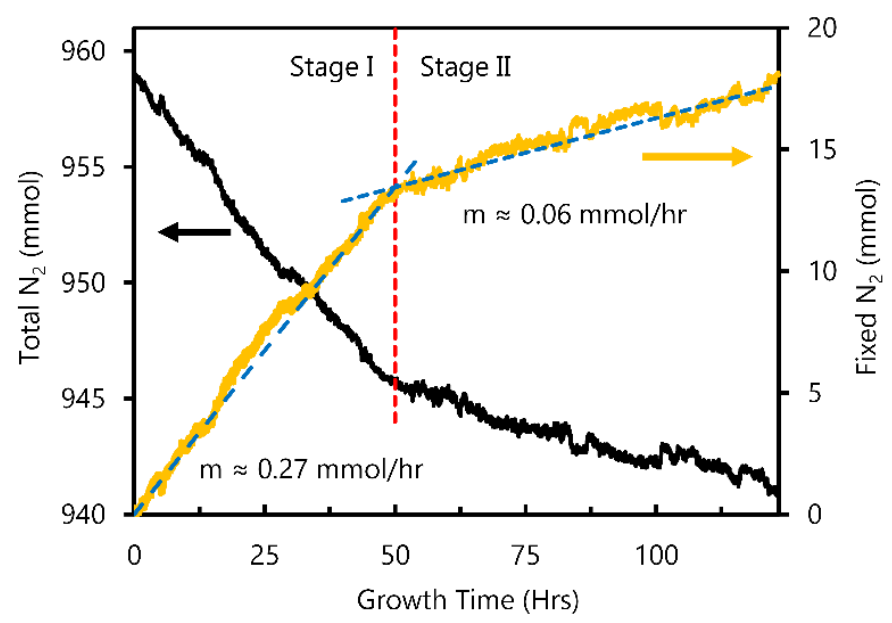

Figure 4. Total $\mathrm{N}_{2}$ decay and cumulative fixed $\mathrm{N}_{2}$ for the growth period, showing two distinct slopes. The fixed $\mathrm{N}_{2}$ curve includes dissolved nitrogen, nitrogen precipitated as solid $G a N$ and any nitrogen taken up by internal reactor or capsule components.

Figure 4 shows the calculated total moles of $\mathrm{N}_{2}$ in the reactor along with the moles of $\mathrm{N}_{2}$ "fixed" or adsorbed from the gas phase over the growth period of $\sim 123$ hours. The fixed $\mathrm{N}_{2}$ curve was obtained by subtracting subsequent values from the peak value of the total (molar) $\mathrm{N}_{2}$ curve, which was arbitrarily chosen as the beginning of the growth period (at 15 hours of total run time). The fixed $\mathrm{N}_{2}$ curve thus reflects dissolved nitrogen, nitrogen precipitated as solid GaN and any nitrogen taken up by internal reactor or capsule components. The last category was negligible, as little to no nitridation of the molybdenum capsule occurred under these conditions based on post-run weight measurements. To refine the estimates for effective temperature and volume, the fixed $\mathrm{N}_{2}$ curve was reconciled to the total mass of GaN grown as measured after the run by adjusting the value of $g$ in calculating the effective temperature. However, this determination of $g$ was bounded by 
also reconciling the total moles of $\mathrm{N}_{2}$ in the reactor at the onset of growth (15 hours of run time) to the amount of $\mathrm{N}_{2}$ filled prior to heating, where $T^{\mathrm{Eff}}=T^{\text {Head }}=T^{\text {Melt }}$.

Using the reconciled values for free volume and effective gas temperature, the fixed $\mathrm{N}_{2}$ from the atmosphere was $\sim 18 \mathrm{mmol}$ by the end of the growth period, though the amount attributed to total GaN formation (seeded growth and PolyGaN) was at most $17 \mathrm{mmol}(90 \%$ of the initial $38 \mathrm{mmol}$ of gallium, or $34 \mathrm{mmol}$ of $\mathrm{GaN}$ ). This $\sim 1 \mathrm{mmol}$ discrepancy may have been due to residual dissolved nitrogen in the melt, nitrogen desorbed onto the reactor internal components, or measurement or estimation error. In any case, any error would mainly affect the absolute value of the fixed $\mathrm{N}_{2}$ curve, with only a minor effect on the slope.

Two distinct slopes are evident in Figure 4, with a transition at $~ 50$ hours of growth time, which implies two distinct regimes (Stage I and Stage II, respectively) of $\mathrm{N}_{2}$ dissolution into the melt and potentially two distinct GaN crystal growth regimes. Stage I occurred from 0-50 hours of growth time and $\sim 13 \mathrm{mmol}$ of $\mathrm{N}_{2}$ were fixed during this period. Stage II occurred from $\sim 50$ to 123 hours of growth time with $\sim 5 \mathrm{mmol}$ of $\mathrm{N}_{2}$ fixed from the reactor atmosphere.

Previous reports indicated that an incubation period between 12 and 50[28] hours is necessary to saturate the melt with nitrogen before $\mathrm{GaN}$ growth commences. The time likely varies due to factors such as melt surface area, melt volume, $\mathrm{N}_{2}$ pressure, etc. Since the end of the incubation period is just before growth commences, it should correspond to the maximum nitrogen concentration in the melt. It is not plausible that Stage I corresponds to the incubation period for our system, since the implied nitrogen solubility is $>10 \mathrm{~mol} \%$ in this case, much greater than any known solubility of nitrogen in sodium-gallium melts. A more reasonable explanation is that incubation took place during the first 15 hours of the run, prior to the peak in pressure, and growth commenced soon thereafter.

The amount of observed seeded GaN growth was $\sim 25 \mathrm{mmol}(\sim 12.5 \mathrm{mmol} \mathrm{N} 2)$, while $\sim 9 \mathrm{mmol}$ of PolyGaN ( 4.5 mmol N ) were found after the run. Based on this, we assume that Stage I corresponded to seeded growth only while Stage II corresponded to PolyGaN growth with little to no seeded growth.

If the absolute nitrogen solubility is indeed as low as expected, the crystal growth curve must largely follow the fixed $\mathrm{N}_{2}$ behavior. This is because the net amount of nitrogen in the melt is given by the difference between the fixed $\mathrm{N}_{2}$ curve and a hypothetical crystal growth curve which traverses the same range of $\sim 17 \mathrm{mmol} \mathrm{N}_{2}$ ( $34 \mathrm{mmol}$ atomic $\mathrm{N}$ or $\mathrm{GaN}$ ) over the same time period, as illustrated schematically in Figure 5. Note that the particular shape of the hypothetical growth curve is entirely arbitrary - only the endpoints are known. 


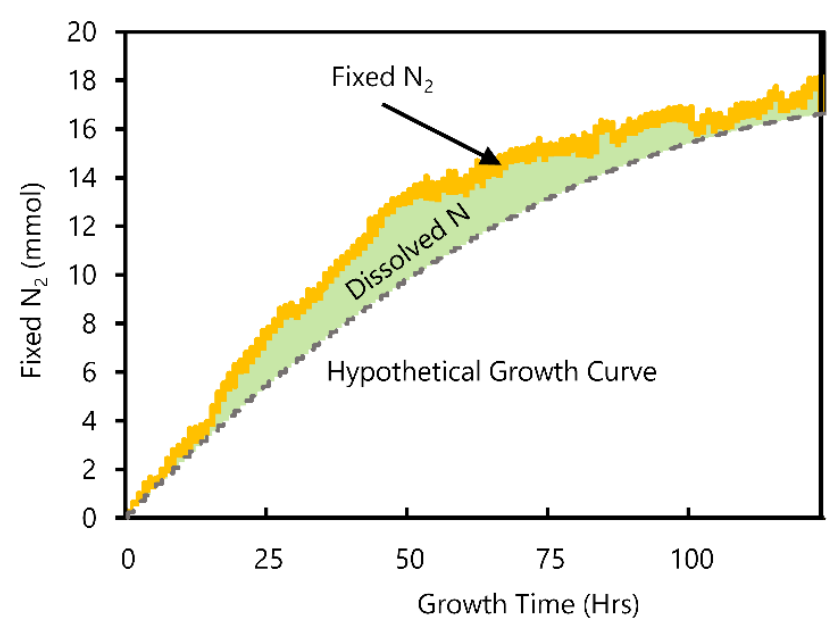

Figure 5. Fixed $N_{2}$ curve from Figure 4 with superimposed hypothetical growth curve. Dissolved nitrogen is represented by the difference between the curves.

For instance, assuming a maximum atomic nitrogen solubility of $\sim 10^{-2}$ at $\%$,[29] the melt could have only accommodated a $\sim 1 \mathrm{mmol}$ of $\mathrm{N}_{2}$ at any particular time. If the solubility was substantially greater, then the deviation between the hypothetical growth curve and fixed $\mathrm{N}_{2}$ curve could of course be larger.

Assuming the growth curve was essentially the same as the fixed $\mathrm{N}_{2}$ curve and the growth area was constant, the actual Stage I seeded growth rate was significantly greater than previously thought, or in excess of $\sim 75 \mu \mathrm{m} / \mathrm{hr}$, based on the grown crystal area and a fixed $\mathrm{N}_{2}$ slope of $0.27 \mathrm{mmol} / \mathrm{hr}$. A growth rate between $\sim 75$ and $90 \mu \mathrm{m} / \mathrm{hr}$ for 50 hours also agrees with a measured grown thickness between 3.7 and $4.5 \mathrm{~mm}$.

Based on $13 \mathrm{mmol}$ of fixed $\mathrm{N}_{2}$ after 50 hours, the residual gallium concentration in melt at the transition between Stage I and Stage II was approximately $13 \mathrm{~mol} \%$, depending on the magnitude of the sodium "offset" and the loss of gallium alloyed with the nickel gasket. Since the slope of the fixed $\mathrm{N}_{2}$ curve was essentially constant, the effect of melt composition on growth rate must be negligible over this range (19 to $13 \mathrm{~mol} \% \mathrm{Ga}$ ). This implies either the solubility change over this range is also small, or else the growth was not mass-transport limited, since the mass transport should be closely related to the absolute amount of nitrogen in the melt.[25,30] The solubility of nitrogen in sodiumgallium melts has been reported to peak near $\sim 20 \mathrm{~mol} \% \mathrm{Ga}$, but the exact behavior with composition is unknown for mixtures more dilute in gallium.[26, 31]

Two challenges prevent us from using these data to identify the rate limiting step and further elucidate the $\mathrm{GaN}$ crystal growth dynamics. First, the expected changes due to dissolved nitrogen are on the order of the noise of our current system, $\sim 1 \mathrm{mmol}$ of $\mathrm{N}_{2}$, and cannot be resolved accurately. This could be overcome with a larger melt volume or higher absolute solubility, i.e., growth at higher pressures or temperatures.[29] Secondly, there is no way to reconstruct the crystal growth curve without the use of other in situ measurements,[32] interrupted growths, growth markers,[33, 34] etc. If these problems can be surmounted, then accurate assessment of the deviation between the fixed $\mathrm{N}_{2}$ 
and crystal growth curve would allow determination of the rate determining step as well as enable calculation of the solubility curve from in situ data.

As another example of the growth information which can be extracted from the pressure decay curve, Figure 6 shows a curve from a separate (not reported in Table 1) run conducted in the same fashion as before which exhibited a large amount of gross $\mathrm{N}_{2}$ leakage. For this run, the peak pressure of $4.94 \mathrm{MPa} \mathrm{N} 2$ decreased to $4.57 \mathrm{MPa}$ over the course of the 65 hours at $860^{\circ} \mathrm{C}$. Once again, the pressure curve was converted to a total $\mathrm{N}_{2}$ decay curve using the same estimations for free volume and effective temperature. For the sake of clarity, $\mathrm{N}_{2}$ leakage was modeled as an exponential decay and subtracted from the fixed $\mathrm{N}_{2}$ curve. The value of the leakage was chosen to reconcile the fixed $\mathrm{N}_{2}$ curve with the observed $\sim 100 \%$ GaN formation from gallium for this run, as only a small amount of gallium was found in the nickel gasket post-run. This approach likely underestimated the fixed $\mathrm{N}_{2}$ slightly, as it didn't account for any residual dissolved nitrogen or nitrogen absorbed elsewhere in the reactor. Error in either the estimation of the total $\mathrm{N}_{2}$ curve or the leakage rate would mainly affect the absolute value rather than the shape or slope of the fixed $\mathrm{N}_{2}$ curve.
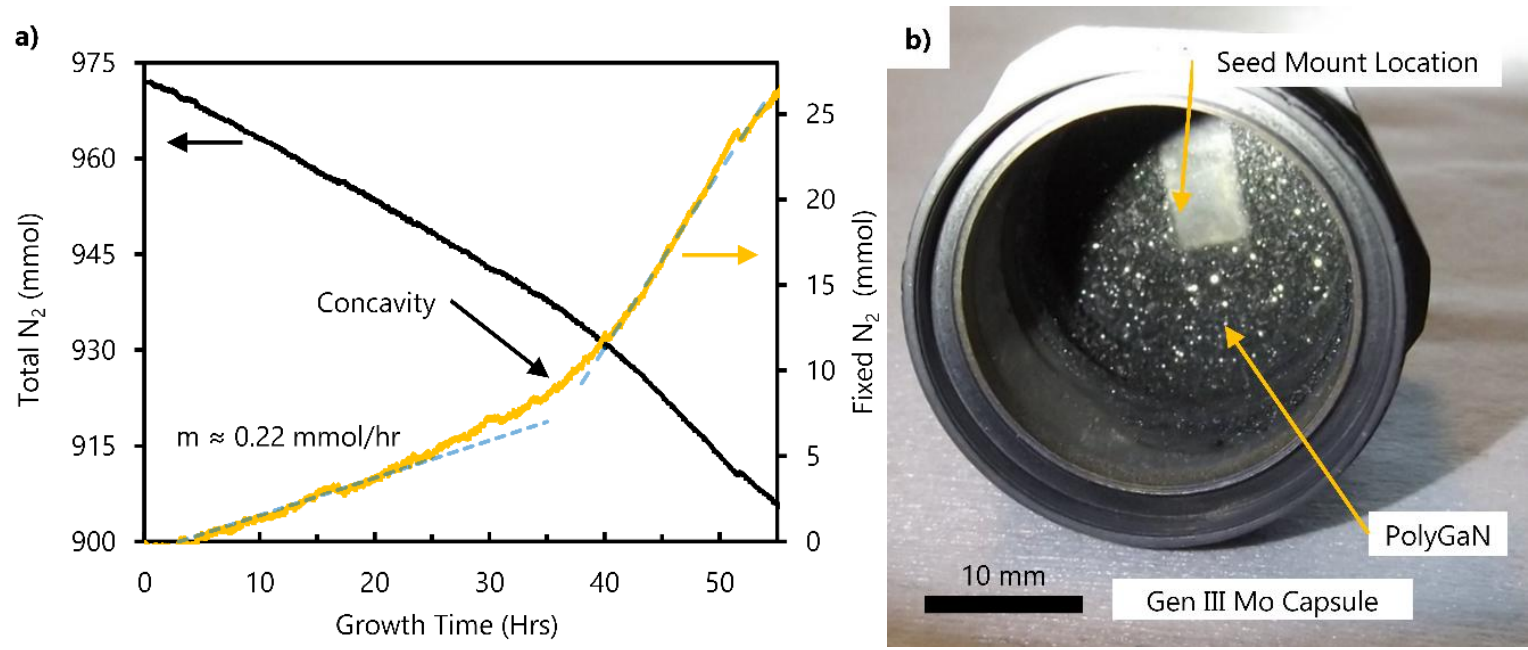

Figure 6. a) Behavior of total $\mathrm{N}_{2}$ and fixed $\mathrm{N}_{2}$ for run with PolyGaN "bloom" showing curvature indicative of PolyGaN nucleation. b) Post-run picture of capsule from run in Figure 6a showing large amounts of PolyGaN nucleated on capsule bottom and sidewalls. The clear area on bottom corresponds to the seed mount location resting on the bottom of the crucible.

The molar decay and fixed $\mathrm{N}_{2}$ curves in Figure 6a show two intervals with fairly constant slopes; from 5-25 hours and 42-52 hours of growth time, respectively. Concavity is evident between these two stages, as well as at the end of the run. Examination after the run showed seeded GaN growth along with a large amount of PolyGaN deposited on the capsule interior walls and bottom along, or a so-called PolyGaN "bloom". The PolyGaN bloom is evident in a picture of the capsule taken after the run, shown in Figure 6b. We have often observed PolyGaN "blooms" of this sort when the reactor $\mathrm{N}_{2}$ leakage rate is high.

Based on the curve shown in Figure 6a, the total $\mathrm{N}_{2}$ fixed during the period between 5 and 25 hours was $\sim 5 \mathrm{mmol}$ of $\mathrm{N}_{2}$, which agrees well with seeded GaN crystal growth of $\sim 9 \mathrm{mmol}$ (4.5 mmol 
of $\mathrm{N}_{2}$ ), as measured by post-run mass change. This implies that single crystal growth largely terminated at the onset of the concavity, and the remainder of the gallium precipitated as PolyGaN. Post-run examination showed no evidence of PolyGaN formation on the grown crystal, which is consistent with the end of single crystal growth after $\sim 25$ hours.

Using the area of the grown crystal, the slope of $\sim 0.22 \mathrm{mmol} / \mathrm{hr}$ implies a growth rate of $\sim 85$ $\mu \mathrm{m} / \mathrm{hr}$ for the duration of the 20 hour period. This result corresponds reasonably well with the measured grown thickness of $\sim 1.63 \mathrm{~mm}$ as well as the proposed termination of single crystal growth at the onset of concavity in the fixed $\mathrm{N}_{2}$ curve. Based on these observations, it is likely the fixed $\mathrm{N}_{2}$ curve once again closely matched the actual GaN crystal growth.

We propose that the concavity in the fixed $\mathrm{N}_{2}$ curve between 25 and 42 hours is characteristic of PolyGaN nucleation, as we have observed this correlation in other runs. The constant-slope interval between 42 and 52 hours therefore represented further growth of the nucleated PolyGaN. The increased slope was presumably due to the widespread nature of the PolyGaN nucleation and resulting increased $\mathrm{N}_{2}$ fixation rate. Finally, the concavity near the end of the growth period was likely due to tapering in the growth rate from further gallium depletion.

\section{Conclusions}

We observed an average GaN crystal growth rate $>50 \mu \mathrm{m} / \mathrm{hr}$ in the $+c$-direction, which is the highest to date for the sodium flux method. It was not clear if the multiple peaks evident in the $\omega$ $\mathrm{XRC}$ or the high oxygen concentrations were linked to the faster growth rate, i.e., if the fast growth occurred at the expense of crystal quality. The SIMS results also indicated rather high concentrations of molybdenum $\left(>10^{19}\right.$ atoms $\left./ \mathrm{cm}^{3}\right)$, which likely leached into the melt from the molybdenum capsule.

We developed a technique based on monitoring of the $\mathrm{N}_{2}$ pressure decay curve to better understand the in situ growth dynamics. Based on analysis of the molar fixed $\mathrm{N}_{2}$ curve, the growth rate for this run was as large as $\sim 90 \mu \mathrm{m} / \mathrm{hr}$. We were unable to identify the rate-determining step, but it appeared unlikely that the growth was mass transport limited. We also used the pressure decay monitoring to identify a characteristic signal for large-scale PolyGaN formation during the course of the run.

Since little optimization of the new system has yet been attempted, it is likely the crystal quality and purity can be improved significantly. Likewise, in situ monitoring of nitrogen fixation during sodium flux growth of GaN will likely yield new insights and improvements to the process.

\section{Acknowledgements}

Thanks to Guy Patterson and Doug Rehn for help with many aspects of equipment design and fabrication. Thanks to Dr. Tom Mates for performing the SIMS measurements and to Steven Griffiths and Thomas Malkowski for many helpful conversations and insights. The authors acknowledge the support from the Solid State Lighting and Display/Energy Center at, University of California, Santa 
Barbara and the Materials Research Laboratory (MRL) Central Facilities, which are supported by the MRSEC Program of the NSF under Award no. DMR 1121053; a member of the NSF-funded Materials Research Facilities Network (www.mrfn.org). This work was supported by the KACST-KAUSTUCSB Solid State Lighting Program.

\section{References}

1. Imade, M., et al., Growth of Large GaN Single Crystals on High-Quality GaN Seed by Carbon-Added Na Flux Method. Appled Physics Express, 3(7): p. 075501. (2010)

2. Imade, M., et al., Growth of Bulk GaN Crystals by the Na-Flux Point Seed Technique. Japanese Journal of Applied Physics, 53(5S1): p. 05FA06. (2014)

3. Fujito, K., et al., Bulk GaN Crystals Grown by HVPE. Journal of Crystal Growth, 311(10): p. 30113014. (2009)

4. Dwiliński, R., et al., Ammonothermal GaN Substrates: Growth Accomplishments and Applications. Physica Status Solidi (a), 207(7): p. 1489-1493. (2011)

5. Mikawa, Y., et al. Ammonothermal Growth of Polar and Non-Polar Bulk GaN Crystal. in Conference on Gallium Nitride Materials and Devices X. San Francisco, CA: International Society for Optics and Photonics. (2015)

6. Yamane, H., et al., Preparation of GaN Single Crystals Using a Na Flux. Chemistry of Materials, 9(2): p. 413-416. (1997)

7. Kawamura, T., et al., First-Principles Investigation of the GaN Growth Process in Carbon-Added Na-Flux Method. physica status solidi (b), 252(5): p. 1084-1088. (2015)

8. de Jong, A.E.F., et al., A Sample Chamber for in Situ High-Energy X-ray Studies of Crystal Growth at Deeply Buried Interfaces in Harsh Environments. Journal of Crystal Growth, 420: p. 84-89. (2015)

9. Kawamura, T., et al., Mechanism for Enhanced Single-Crystal GaN Growth in the C-Assisted NaFlux Method. Applied Physics Express, 9(1): p. 015601. (2016)

10. Koukitu, A., et al., Thermodynamic Analysis of Hydride Vapor Phase Epitaxy of GaN. Japanese Journal of Applied Physics, 37(part 1): p. 762-765. (1998)

11. Kumagai, Y., et al., Thermodynamics on Tri-Halide Vapor-Phase Epitaxy of GaN and $\operatorname{In}(x) G a(1-x) N$ Using GaCl3 and InCl3. Journal of Crystal Growth, 231(1): p. 57-67. (2001)

12. Steigerwald, T.G., et al., Feasibility of Density and Viscosity Measurements under Ammonothermal Conditions. Journal of Crystal Growth, 403: p. 59-65. (2014)

13. Pimputkar, S. and S. Nakamura, Decomposition of Supercritical Ammonia and Modeling of Supercritical Ammonia-Nitrogen-Hydrogen Solutions with Applicability toward Ammonothermal Conditions. The Journal of Supercritical Fluids, 107: p. 17-30. (2016)

14. Zhang, S., et al., Intermediates in Ammonothermal GaN Crystal Growth under Ammonoacidic Conditions. European Journal of Inorganic Chemistry, 2013(31): p. 5387-5399. (2013)

15. Pendurti, S., Q.-S. Chen, and V. Prasad, Modeling Ammonothermal Growth of GaN Single Crystals: The Role of Transport. Journal of Crystal Growth, 296(2): p. 150-158. (2006)

16. Chen, Q.-S., et al., Modeling on Ammonothermal Growth of GaN Semiconductor Crystals. Progress in Crystal Growth and Characterization of Materials, 58(2): p. 61-73. (2012) 
17. Mori, Y., et al., Growth of Bulk GaN Crystal by Na Flux Method under Various Conditions. Journal of Crystal Growth, 350(1): p. 72-74. (2012)

18. Imade, M., et al., Centimeter-Sized Bulk GaN Single Crystals Grown by the Na-Flux Method with a Necking Technique. Crystal Growth \& Design, 12(7): p. 3799-3805. (2012)

19. Yoshida, T., et al., Ultrahigh-Speed Growth of GaN by Hydride Vapor Phase Epitaxy. physica status solidi (c), 8(7-8): p. 2110-2112. (2011)

20. Ehrentraut, D., et al., High Quality, Low Cost Ammonothermal Bulk GaN Substrates. Japanese Journal of Applied Physics, 52(8S): p. 08JA01. (2013)

21. Von Dollen, P., et al., A New System for Sodium Flux Growth of Bulk GaN Part I: System Design. Journal of Crystal Growth. (submitted)

22. Wilson, R.G., SIMS Quantification in Si, GaAs, and Diamond-an Update. International Journal of Mass Spectrometry and Ion Processes, 143: p. 43-49. (1995)

23. Konishi, Y., et al., Growth of Prismatic GaN Single Crystals with High Transparency on Small GaN Seed Crystals by Ca-Li-Added Na Flux Method. Applied Physics Express, 5: p. 025503. (2012)

24. Murakami, K., et al., Effects of Solution Stirring on the Growth of Bulk GaN Single Crystals by Na Flux Method. Japanese Journal of Applied Physics, 52: p. 08JA03. (2013)

25. Morishita, M., et al., The Influences of Supersaturation on LPE Growth of GaN Single Crystals Using the Na Flux Method. Journal of Crystal Growth, 270(3-4): p. 402-408. (2004)

26. Mori, Y., et al., Growth of GaN Crystals by Na Flux Method. ECS Journal of Solid State Science and Technology, 2(8): p. N3068-N3071. (2013)

27. Okamoto, H., Ga-Ni (Gallium-Nickel). Journal of Phase Equilibria and Diffusion, 31(6): p. 575576. (2008)

28. Imabayashi, H., et al., Growth and Evaluation of Bulk GaN Crystals Grown on a Point Seed Crystal by Ba-Added Na Flux Method. Sensors and Materials, 25(3): p. 165-176. (2013)

29. Kawamura, F., et al., Study of the Metastable Region in the Growth of GaN Using the Na Flux Method. Journal of Crystal Growth, 311(22): p. 4647-4651. (2009)

30. Scheel, H. and D. Elwell, Crystal Growth from High-Temperature Solutions. Online ed.: Academic Press. ISBN: (2011)

31. Kawahara, M., et al., A First-Principles Investigation on the Mechanism of Nitrogen Dissolution in the Na Flux Method. Journal of Applied Physics, 101(6): p. 066106. (2007)

32. Sun, G., et al., Study on the Kinetics of the Formation Reaction of GaN from Ga-Solutions under Ammonia Atmosphere. Journal of Crystal Growth, 305(2): p. 326-334. (2007)

33. Damen, J.P.M. and J.M. Robertson, Induced Non-Periodic Growth Striations in Flux-Grown Magnetic Oxide Single-Crystals. Journal of Crystal Growth, 16(1): p. 50-53. (1972)

34. Kim, K.M., A.F. Witt, and H.C. Gatos, Crystal-Growth from Melt under Destabilizing Thermal Gradients. Journal of the Electrochemical Society, 119(9): p. 1218-1226. (1972) 